\title{
Alopecia areata and salmon patch: an actual association?
}

\author{
Daniel Fernandes Melo ${ }^{1 凶}$, Violeta Duarte Tortelly ${ }^{1}$, Taynara de Mattos Barreto ${ }^{2}$, Elaine de Souza Albernaz ${ }^{3}$
}

Keywords: alopecia, alopecia areata, hair disease, skin diseases, vascular

Received: 19 September 2017 | Returned for modification: 19 November 2017 | Accepted: 17 December 2017

\section{To the Editor}

Alopecia areata is a chronic disease that affects hair follicles. Its etiology remains unknown, but it is believed to have a multifactorial origin with autoimmune and genetic components. The disease affects both sexes and all ages. The classic clinical presentation is characterized by an asymptomatic, smooth surfaced alopecic area with normal colored skin, more often on the scalp but sometimes on other hairy areas of the body. The hair pull test is usually positive at the periphery of the patch when the disease is active, and trichoscopy is a useful tool for diagnosis and follow-up. The ophiasis, totalis, and universalis forms are rarer and have worse prognoses (1).

Extra-follicular affections, including nail and ocular abnormalities, are classically related to a worse prognosis of the disease, but some authors have wondered whether the presence of salmon patch on the nape of the neck could also indicate a more severe evolution of the condition (1).

Salmon patch is a congenital venous malformation that predominantly affects the midline of the body. It may also be called nevus flammeus (neonatorum) or nevus simplex. Clinically, it presents as irregular pink or reddish macules that may or may not be confluent, fade under pressure, and become more visible during hard crying, fever, or temperature changes (2). Its prevalence is an estimated 30 to $40 \%$ of all newborns, with a slightly higher rate in female newborns than in male $(2,3)$. The sites most commonly affected are the nape of the neck, the glabella, and the eyelids; however, multiple sites can be affected. Salmon patch is usually transitory, disappearing within the first 2 years of life. However, almost half of the lesions located in the nape or sacrum, and a small proportion of those located in the glabella, persist (2).

Infantile lesions usually do not present with histopathological changes, but persistent salmon patch in adults shows ectasia of the subpapillary vessels, and perivascular inflammatory infiltrate may be associated with these lesions when they are seen in alopecia areata patients $(3,4)$.

Although salmon patch is a benign condition that does not require treatment, it should be differentiated from other lesions that may require therapeutic intervention, such as port wine stains and infant hemangiomas. However, it can be associated with other diseases in rare cases, and previous studies have observed a possible relation between salmon patch in the nape of the neck and the chronic form or more extensive forms of alopecia areata (2-5).

In 1988, Hatzis et al. demonstrated a significant increase in the incidence of salmon patch in patients with more severe forms of alopecia areata-such as ophiasis, totalis, and universalis-compared to the general population, suggesting that the presence of the lesion could be a useful marker of widespread and chronic disease. In later studies, the same relationship was observed between a higher prevalence of salmon patch in the neck and the more severe forms of alopecia areata (3-6). Akhyani et al. carried out a study of 199 patients with alopecia areata and 215 controls, with results that evidenced a statistically significant association between the presence of salmon patch and a greater severity and duration of alopecia areata (3).

Aghaei evaluated the efficacy of diphenylcyclopropenone in the treatment of severe alopecia areata, observing that salmon patch was present in $40.7 \%$ of the patients, whereas nail involvement (already known to be associated with a worse prognosis) was seen in $59.3 \%$ and thyroid diseases in $11.1 \%$. The study also evaluated salmon patch as a marker of treatment outcome, finding a significant correlation between the presence of the malformation on the posterior neck and a less favorable treatment response (7).

The exact pathogenesis of this association is still unclear. It has been suggested that the high prevalence of salmon patch in patients with alopecia areata could be a sign of epidermal atrophy allowing ectasic subpapillar vessels in the nape of the neck to become visible $(3,8,9)$. However, the study by Hatzis et al. did not observe signs of epidermal atrophy in the lesions that were submitted to histopathological examination $(3,8)$.

Another study, reporting the case of a patient with total alopecia areata associated with salmon patch on the nape of the neck, showed a sustained repilation in the area of the vascular lesion, even after treatment was withdrawn. It is known that hair loss in alopecia areata may be associated with decreased blood supply and, in the case described, sustained repilation occurred only in the area with salmon patch, where the blood supply and the number of follicles are presumably larger (10).

WenChieh Chen described a universal alopecia areata case that spared the port wine stain area on the scalp. A genetic mosaicism present in the nevus lesions could explain the fact that a melanocytic nevus is spared from the capillary loss caused by alopecia areata, consisting of a type of Renbök phenomenon. However, in the case described the lesion was not melanocytic, but was an injury of vascular origin. This suggests that vascular factors, hitherto unknown, may antagonize the immune aggression that occurs in alopecia areata (11).

The validity of the clinical association between salmon patch and alopecia areata is still uncertain because of alopecia areata's high prevalence in the general population. Additionally, variables such as phototype, density, and hair color can make it difficult to properly evaluate salmon patch (12).

In addition to reports of a link between salmon patch and alopecia areata, we have observed in our practice patients with severe forms of alopecia areata and concomitant salmon patch on the nape of the neck (Figs. 1-2). It is possible that the association represent 
observer bias because the lesions are much more noticeable in the naked occipital region and because the ophiasis form has a worse prognosis. Therefore, comparative studies with a greater number

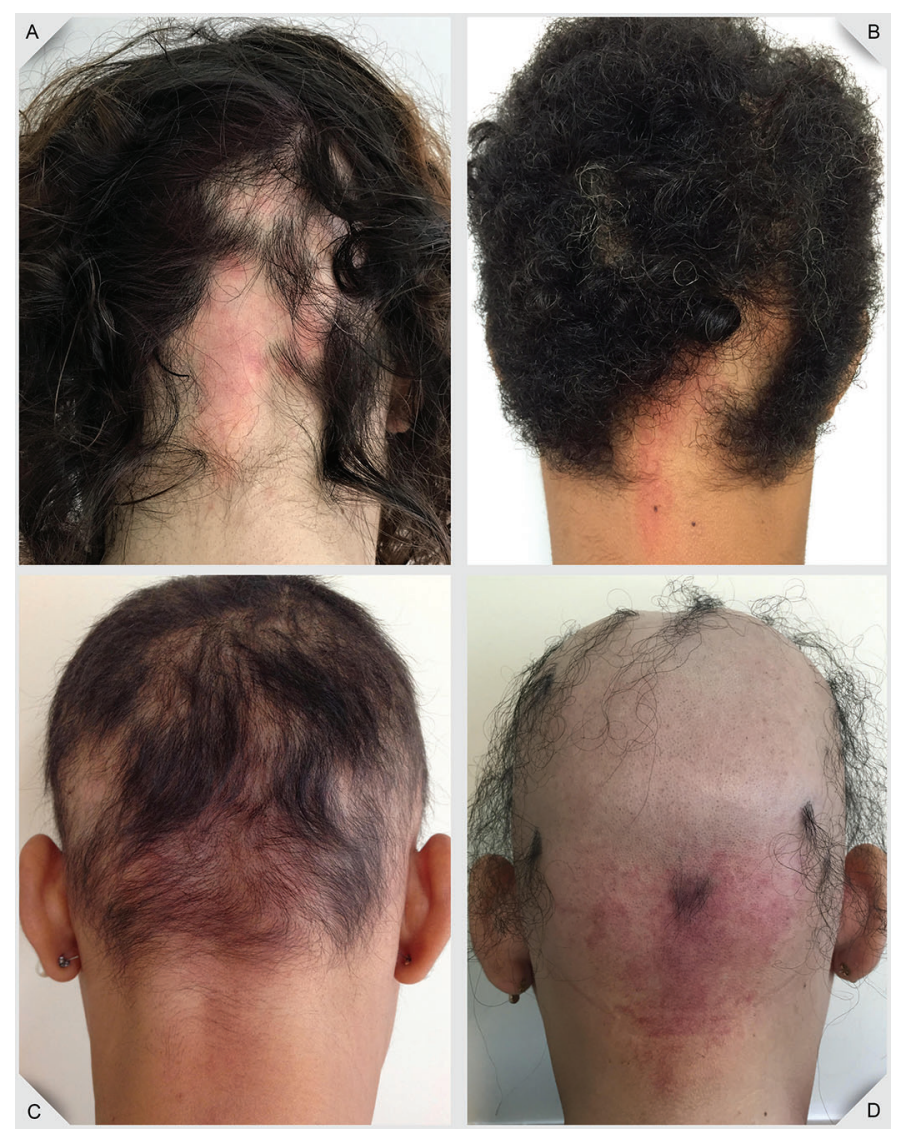

of patients are necessary to validate this association, which-if it is truly significant-could act as a prognostic marker of great value in the evaluation of patients with alopecia areata.

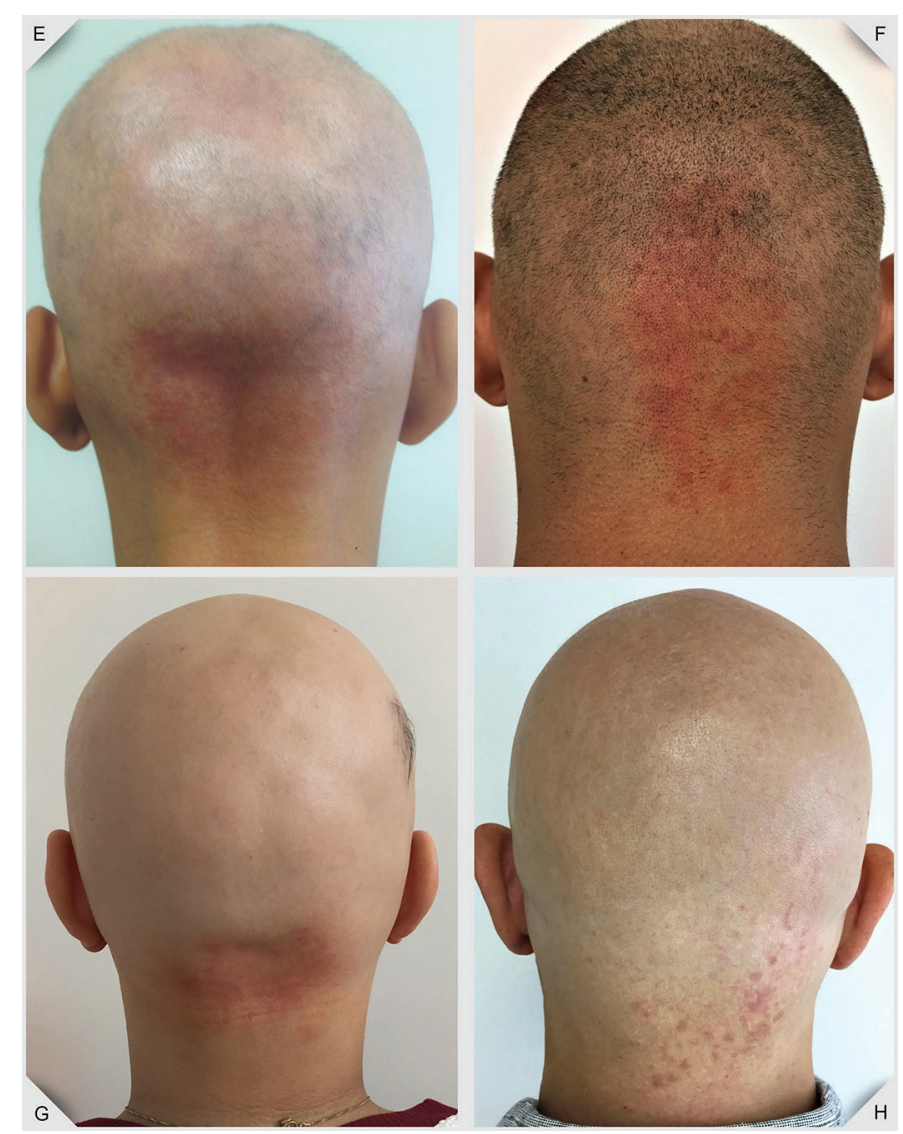

Figures 1 and 2 | Patients presenting with extensive areata alopecia and salmon patch on the neck.

\section{References}

1. Rivitti EA. Alopecia areata: a revision and update. An Bras Dermatol. 2005;80:5768.

2. Monteagudo B, Labandeira J, Acevedo A, Cabanillas M, León-Muiños E, Fernández-Prieto R, et al. Salmon patch: a descriptive study. Actas Dermosifiliogr. 2011;102:24-7.

3. Akhyani M, Farnaghi F, Seirafi H, Nazari R, Mansoori P, Taheri A. The association between nuchal nevus flammeus and alopecia areata: a case-control study. Dermatology. 2005;211:334-7.

4. Hatzis J, Kostakis P, Tosca A, Parissis N, Nicolis G, Varelzidis A, et al. Nuchal nevus flammeus as a skin marker of prognosis in alopecia areata. Dermatologica. 1988;177:149-51.

5. Camacho F, Navas J. Nuchal nevus flammeus in alopecia areata. Dermatology. 1992;184:158.
6. Orecchia G, Perfetti L. Nuchal nevus flammeus and alopecia areata. Dermatologica. 1989;179:93-4.

7. Aghaei S. Topical immunotherapy of severe alopecia areata with diphenylcyclopropenone (DPCP): experience in an Iranian population. BMC Dermatol. 2005;5:6.

8. Hatzis J, Kostakis P, Tosca A, Parissis N, Nicolis G, Varelzidis A, et al. In reply: Nuchal nevus flammeus. Dermatologica. 1989;179:223.

9. Van Baar H, Penet CM, Happle R. Nuchal nevi flammei and alopecia areata. Dermatologica. 1989;179:52-3.

10. Burgoyne JS. Regrowth of hair in nevus flammeus. Arch Dermatol. 1972;105:758.

11. Chen W. Alopecia areata universalis sparing nevus flammeus. Dermatology. 2005;210:227-8.

12. Manzke H. Incidence of nuchal nevus flammeus. Dermatologica. 1989;179:55 